\begin{tabular}{|c|l|}
\hline Title & Liquid-phase diffusivity of benzene within mesoporous materials measured by a laser Raman technique \\
\hline Author(s) & Nakasaka, Y uta; Tago, Teruoki; Y ano, Kazuhisa; Masuda, Takao \\
\hline Citation & $\begin{array}{l}\text { Chemical Engineering Science, 65(1), 226-231 } \\
\text { https://doi.org/10.1016/.Ces.2009.06.022 }\end{array}$ \\
\hline Issue Date & 2010-01-01 \\
\hline Doc URL & http://hdl.handle.net/2115/48285 \\
\hline Type & article (author version) \\
\hline File Information & CES65-1_226-231.pdf \\
\hline
\end{tabular}

Instructions for use 


\title{
Liquid-Phase Diffusivity of Benzene within Mesoporous Materials Measured by a Laser Raman Technique
}

\author{
Yuta Nakasaka $^{\mathrm{a}}$, Teruoki Tago ${ }^{\mathrm{a},{ }^{*}}$, Kazuhisa Yano ${ }^{\mathrm{b}}$, and Takao Masuda \\ a Division of Chemical Process Engineering, Graduate School of Engineering, Hokkaido University, \\ Sapporo 060-8628, Japan \\ ${ }^{\mathrm{b}}$ Toyota Central R\&D Labs., Inc., Nagakute, Aichi 480-1192, Japan \\ * Corresponding author should be addressed \\ TEL/FAX: (+81)-11-706-6551 / (+81)-11-706-6552, E-mail: tago@eng.hokudai.ac.jp
}

Keywords: Intracrystalline diffusivity, Mesoporous silica, Liquid phase, BenzeneCyclohexane solution, Raman spectroscopy 


\begin{abstract}
The intracrystalline diffusivities of benzene within a series of porous materials in the liquid phase (cyclohexane was used as solvent) were measured by a constant volumetric method using Raman spectroscopy at a temperature range from 323 to $393 \mathrm{~K}$. Silicalite-1, mono-dispersed mesoporous silica spheres (MMSS), Silica Gel, $\gamma-\mathrm{Al}_{2} \mathrm{O}_{3}$, and $\mathrm{SiO}_{2}-\mathrm{Al}_{2} \mathrm{O}_{3}$ were used as adsorbents. The intracrystalline diffusivity was calculated by parameter fitting using theoretical equations and the experimental transient change of benzene concentrations with time in response to the adsorption. The intracrystalline diffusivities of benzene within mesoporous silicas were almost the same as that within the micropore of silicalite-1, though the pore diameters of silicalite-1 and mesoporous silicas were different from each other. It is considered that the pore walls as well as the solvent molecules of cyclohexane affect the diffusion of benzene molecules in the meso pore region.
\end{abstract}




\section{Introduction}

Microporous and mesoporous materials are widely used in industrial processes. In catalytic reactions using porous materials such as zeolite, reactant molecules diffuse into the pores of the catalyst from bulk fluid and adsorb on the active sites, where chemical reactions proceed. Accordingly, the activity and selectivity of the reaction are closely related to the intracrystalline diffusivities, which will affect reactor design.

Several studies have measured the hydrocarbon diffusivities within zeolite in the gas phase (Zikánová et al., 1987; Shen and Rees, 1991). In addition to this, several measurement methods, including microscopic (Pulse field gradient NMR (Jobic et al., 1989), quasi-elastic neutron scattering (Xial and Wei, 1992)) and macroscopic methods (the gravimetric method (Shah et al., 1995), the constant volumetric method (Song and Rees, 1996), and Zero-length column technique (Jama et al., 1997)) have been proposed. We have also reported the intracrystalline diffusivity of benzene, toluene, xylene isomers, and lighter hydrocarbons within MFI-type and Y-type zeolite in the gas phase by the constant volumetric method (Masuda et al., 1996, 1998, 2000, 2001; Masuda, 2003a, 2003b; Fujikata et al., 1998). There have been few reports, however, concerning direct experimental measurements of diffusivity under high-temperature and high-pressure conditions in the liquid phase. We have developed a new constant volumetric method for measuring the intracrystalline diffusivity within porous materials in the liquid phase using Raman spectroscopy (Nakasaka et al., 2008).

The pore sizes of porous materials are classified into 3 types: micro pore $(\sim 2 \mathrm{~nm})$, meso pore (2 50nm), and macro pore (50nm ). Synthesis of mesoporous materials with uniform and ordered pores has been investigated (Yokoi et al., 2003; Yano and 
Fukushima, 2003, 2004). In addition, mesoporous materials have been applied to adsorbents and catalysts (Iwamoto et al., 2003) since the discovery of the mesoporous silicas (Yanagisawa et al., 1990; Inagaki et al., 1993; Kresge et al., 1992). For these applications, information regarding the diffusivity and diffusion mechanism within mesoporous materials is necessary. Qiao et al. (2005) have studied the diffusivity of ndecane in MCM-41 silicas with pore diameters between 3.0 and $4.3 \mathrm{~nm}$ in the 303-363 K temperature range by the ZLC technique in the gas phase, and the diffusivity was found to be in the range of $(0.24-9.37) \times 10^{-12} \mathrm{~m}^{2} \mathrm{~s}^{-1}$.

The main objects of this study were to measure the intracrystalline diffusivities of benzene within a series of porous materials in the liquid phase by a constant volumetric method, and the effects of the pore diameter of the adsorbents on the diffusivity were investigated.

\section{Experimental}

\subsection{Adsorbents}

The adsorbents used in this study and these pore diameters are listed in Table 1. Silicalite-1 was used as a microporous material. The silicalite-1 was synthesized hydrothermally at $473 \mathrm{~K}$ for $48 \mathrm{~h}$ from a sodium silicate $\left(\mathrm{Na}_{2} \mathrm{SiO}_{3}\right)$ solution using a tetran-propyl ammonium bromide (TPABr) as a template (Masuda et al., 2003c). The silicalite-1 powder thus prepared was washed with distilled water and then dried in an air atmosphere, followed by calcination at $773 \mathrm{~K}$ to remove the template. To remove the $\mathrm{Na}^{+}$ 
cations remaining within silicalite-1, the silicalite- 1 was treated with $\mathrm{NH}_{4} \mathrm{NO}_{3}$ aqueous solution under reflux conditions. The silicalite- 1 thus obtained was confirmed by X-ray diffraction analysis (XRD; JEOL JDX-8020) to have the same structure as the pentasiltype zeolite. The crystal size of silicalite-1 was measured by field emission scanning electron microscopy (JEOL JSM-6500F). Silicalite-1 was pelletized without any binders, and was crushed and sieved to yield samples with a particle size of 0.3-0.5 mm.

\section{[Table 1]}

Mono-dispersed mesoporous silica spheres with a highly ordered hexagonal regularity (MMSS-10, MMSS-16, MMSS-18, MMSS-22), and Silica Gel (GL Sciences Inc. Intersil SIL-100A) were employed as mesoporous materials. Because these mesoporous materials as well as silialite-1 have main straight channels for diffusion, it is considered that the effect of pore size on the diffusivity can be investigated, regardless of the tortuosity and pore-connecting. Details of the preparation procedure for the monodispersed mesoporous silica spheres have been provided in Yano's previous paper (Yano and Fukushima, 2003, 2004). These materials were also pelletized without any binders, and were crushed and sieved to yield samples with particle sizes of 0.85-1.18 $\mathrm{mm}$ before calcination at $773 \mathrm{~K}$. In addition, $\gamma-\mathrm{Al}_{2} \mathrm{O}_{3}$ and $\mathrm{SiO}_{2}-\mathrm{Al}_{2} \mathrm{O}_{3}$ were used as macroporous materials, and the pore diameters of $\gamma-\mathrm{Al}_{2} \mathrm{O}_{3}$ and $\mathrm{SiO}_{2}-\mathrm{Al}_{2} \mathrm{O}_{3}$ were measured by mercury porosimetry using the Shimadzu Auto Pore 9500. The scanning electron micrographs (SEM) of silicalite-1 and mesoporous silicas (MMSS-10, MMSS-16, MMSS-18 and Silica Gel) are shown in Fig. 1.

\section{[Figure 1]}


2.2. Measurement of intracrystalline diffusivity in the liquid phase by a constant volumetric method

The intracrystalline diffusivity of benzene within the porous materials in cyclohexane solution was measured by the conventional constant volume method using Raman spectroscopy. The transient change in benzene concentrations in the solution due to its adsorption and diffusion within the porous materials are expressed by the following theoretical equations (Crank, 1975) for porous materials with a hexagonal slab shape (MFI type zeolite; Silicalite-1) and a spherical shape (MMSS-10, MMSS-16, MMSS-18, MMSS-22, Silica Gel, $\mathrm{SiO}_{2}-\mathrm{Al}_{2} \mathrm{O}_{3}$ and $\gamma-\mathrm{Al}_{2} \mathrm{O}_{3}$ ), based on the rigorous mass balance measured using the Raman spectroscopy.

[hexagonal slab shape]

$$
\frac{M_{\mathrm{t}}}{M_{\mathrm{e}}}=\frac{C_{0}-C_{t}}{C_{0}-C_{e}}=1-\sum_{n=1}^{\infty} \frac{2 \alpha(1+\alpha)}{1+\alpha+\alpha^{2} q_{n}^{2}} \exp \left(-\frac{D_{a d} q_{n}^{2}}{L^{2}} t\right)
$$

where $\alpha=V /\left(a_{m} W H L\right)$ and $q_{\mathrm{n}}$ are non-zero positive roots of $\tan q_{n}=-\alpha q_{n}$.

[spherical shape]

$$
\left.\frac{M_{\mathrm{t}}}{M_{\mathrm{e}}}=\frac{C_{0}-C_{t}}{C_{0}-C_{e}}=1-\sum_{n=1}^{\infty} \frac{6 \alpha(1+\alpha)}{9+9 \alpha+\alpha^{2} q_{n}^{2}} \exp -\frac{D_{a d} q_{n}^{2}}{R_{0}^{2}} t\right)
$$

where $\alpha=V /\left(a_{m} W H R_{0}\right)$ and $q_{\mathrm{n}}$ are non-zero positive roots of $\tan q_{n}=-\alpha \frac{3 q_{n}}{3+\alpha q_{n}^{2}}$.

$M_{\mathrm{t}}$ is the amount adsorbed at a time $t, M_{\mathrm{e}}$ is the $M_{\mathrm{t}}$ value at equilibrium, and $D_{\text {ad }}$ is the intracrystalline diffusivity of benzene in the adsorption process. $C_{\mathrm{t}}$ is the benzene concentrations in cyclohexane solution at a time $t$, and $C_{0}$ and $C_{\mathrm{e}}$ are the $C_{\mathrm{t}}$ values at 
initial and equilibrium, respectively. $L$ and $R_{0}$ are the half-thickness of the hexagonal slab and spherical shapes, respectively, and $a_{\mathrm{m}}$ is the outer surface area of the crystal. $W$ and $V$ are the weight of the adsorbent and the volume of the liquid phase, respectively. $H$ is the partition factor.

Using the change in the benzene concentration in the solution with time and these equations, the intracrystalline diffusivity was calculated (an example of the change in the benzene concentration in cyclohexane is indicated in Fig. 2). The details of the apparatus and procedure are the same as those in our previous work (Nakasaka et al., 2008).

\section{[Figure 2]}

\section{Results and Discussion}

3.1 Effects of pellet size on the diffusivities within mesoporous silicas

Benzene molecules diffuse in cyclohexane solution in accordance with the molecular diffusion mechanism, followed by adsorption and diffusion in the pores of porous materials. In the diffusion and adsorption of benzene within the adsorbent, it is considered that there are three mass-transfer resistances: (1) diffusion across the laminar film around the interface between the bulk solution and the adsorbent surface; (2) diffusion through the macro pores among the adsorbent crystals and/or particles; and (3) diffusion in the micro or meso pores within the adsorbent. In our previous paper (Nakasaka, 2008), it was found that the mass-transfer resistances across the laminar film 
and through macro pores of pelletized silicalite-1 are negligible small. In this study, mesoporous silicas were used, and the pore diameter was much larger than the micro pore of silicalite-1. When the diffusion velocity of benzene within the meso pores is faster than that within macro pores among adsorbents, the diffusion velocity of benzene within the macro pore becomes the rate-limiting step of the mass transfer. Therefore, the measurement of the intracrystalline diffusivity is required to be conducted under the condition that the diffusion velocity within the meso pores $\left(D_{\mathrm{ad}} / R_{0}{ }^{2}\right)$ is smaller than that within the macro pores $\left(D_{\text {macro }} / r^{2}\right)$, as expressed by the next equation:

$$
\frac{D_{\text {macro }}}{r^{2}}>\frac{D_{a d}}{R_{0}^{2}}
$$

where, $D_{\text {macro }}$ and $r$ are the diffusivity within the macro pore and the half size of secondary particle (pelletized crystal), respectively.

Figure 3 shows the effects of the pellet size of MMSS-16 on the intracrystalline diffusivity of benzene within MMSS-16 at 323K. The intracrystalline diffusivities of benzene were independent of the pellet size in this range, and it is considered that the mass-transfer resistances through the macro pore are negligibly small. The calculated maximum pellet size of MMSS-16 derived from equation 3 was approximately $5 \mathrm{~mm}$.

\section{[Figure 3]}

\subsection{Effects of the amount of adsorbed benzene on the intracrystalline diffusivity}

In this method, the initial as well as equilibrium concentrations of benzene can be directly obtained from the concentration changes with time shown in Fig. 2. Using the concentrations, the diffusion molecule concentrations in solution and the amount of 
diffusion molecule adsorbed onto adsorbents at the equilibrium state can be calculated. Figure 4 shows typical adsorption isotherms of benzene onto the mesoporous materials at 323K. The linear relationship between the amounts of benzene adsorbed onto mesoporous silicas and the equilibrium benzene concentration indicate that the adsorption isotherm can be expressed by a Henry-type equation:

$$
q_{\mathrm{e}}=K C_{\mathrm{e}}
$$

where $q_{\mathrm{e}}$ and $C_{\mathrm{e}}$ are the amount of adsorbed diffusion molecule and the diffusion molecule concentration in the solution, respectively, at the equilibrium condition.

\section{[Figure 4]}

Figure 5 shows the typical dependence of the intracrystalline diffusivity of benzene on the amount of adsorbed benzene within mesoporous silicas. The measured diffusivity exhibited an almost constant value, regardless of the different amounts of adsorbed benzene within mesoporous silicas. This result indicates that the intracrystalline diffusivities of benzene within mesoporous silicas are independent of the amount of benzene adsorbed within mesoporous silicas.

\section{[Figure5]}

3.3 Intracrystalline diffusivities of benzene within a series of porous materials

Figure 6 shows the typical Arrhenius plots of intracrystalline diffusivities of benzene, which represent the mobility of benzene, within the porous materials used here. The intracrystalline diffusivity of benzene within silicalite-1 in the gas phase is also 
shown in this figure for comparison (Nakasaka et al., 2008). The intracrystalline diffusivity of benzene within silicalite- 1 in cyclohexane solution at $323 \mathrm{~K}$ was $1.2 \times 10^{-17}$ $\mathrm{m}^{2} \mathrm{~s}^{-1}$. In contrast, the intracrystalline diffusivity of benzene within mesoporous silicas of MMSS-10, MMSS-16, MMSS-18, and MMSS-22 in cyclohexane solution were $8.3 \times 10^{-18}$, $5.9 \times 10^{-18}, 2.0 \times 10^{-17}$, and $2.1 \times 10^{-17} \mathrm{~m}^{2} \mathrm{~s}^{-1}$, respectively. Though the pore diameters of the mesoporous silicas shown in Fig. 6 are approximately 4 times larger than molecular diameter of benzene, the intracrystalline diffusivities of benzene within mesoporous silicas in liquid phase are almost the equal to and/or smaller than that of silicalite-1. Moreover, the intracrystalline diffusivities of benzene within Silica Gel, $\gamma-\mathrm{Al}_{2} \mathrm{O}_{3}$ and $\mathrm{SiO}_{2}-\mathrm{Al}_{2} \mathrm{O}_{3}$ were $1.2 \times 10^{-15}, 4.2 \times 10^{-10}$, and $1.5 \times 10^{-10}$, respectively, and the intracrystalline diffusivities of benzene within macroporous materials were $10^{7}$ times larger than those within mesoporous silicas. Based on these results, the relationship between pore diameter and the intracrystalline diffusivity of benzene at $323 \mathrm{~K}$ and the activation energy of intracrystalline diffusivities of benzene within the series of adsorbents are summarized in Fig. 7. Using the relationship indicated in this figure, the intracrystalline diffusivity of benzene can be predicted within porous silicas in the liquid phase.

\section{[Figures 6 and 7]}

The diffusivities of benzene within mesoporous silicas were almost the same and/or slightly smaller than those within the micropore region, and the values increased with increasing pore diameter. In contrast, the activation energy of diffusion in the micropore region was much higher than those in the mesopore and macropore regions. Moreover, the activation energies in the mesopore and macropore regions were 
independent of the pore diameter. Accordingly, it is considered that benzene and cyclohexane molecules adsorbed onto the pore wall of mesoporous silica decrease the effective pore diameter where benzene molecules can diffuse, leading to a small diffusivity. Moreover, since the diffusion molecules within mesopores are affected by the adsorbed solvent molecules on the pore walls, the activation energy of diffusion in the mesopore region shows almost the same value as that in the macropore region.

It is known that the diffusivities of molecules in the gas phase are classified by the relationship between the mean free path and the radius of diffusion space (Hashimoto, 1979). The classified regions in the gas phase are a configurational diffusion region, a Knudsen diffusion region, a transition region, and a molecular diffusion region. In liquidphase diffusion, it is considered that the diffusion mechanism can also be classified based on the relationship between pore diameter and molecular size, in the same manner as in the gas phase, though the solvent molecules exist in the pore. The classified regions in the liquid phase are (1) a configurational diffusion region, (2) a transition region, and (3) a molecular diffusion region: the findings are summarized in Fig. 8.

\section{[Figure 8]}

\section{Conclusion}

Using the constant volume method, the intracrystalline diffusivities of benzene within a series of porous solid materials in the liquid phase (cyclohexane used as a solvent) were measured using a Raman spectrophotometer in the temperature range of 323-393 K. In the liquid phase, benzene molecules as well as cyclohexane were adsorbed 
onto the pore walls of meso-porous materials, leading to a decrease in the effective pore diameter for diffusion. It was considered that these adsorbed molecules on the pore wall affected the intracrystalline diffusivity of benzene. Based on the dependence of the intracrystalline diffusivities and their activation energies on the pore size, the diffusion mechanism in the liquid phase can be classified according to regions: (1) a configurational diffusion region; (2) a transition region; and (3) a molecular diffusion region.

\section{Acknowledgements}

This work was supported in part by the Global COE Program (Project No. B01: Catalysis as the Basis for Innovation in Materials Science) from the Ministry of Education, Culture, Sports, Science and Technology, Japan, and by the Industrial Technology Research Grant Program in 2006, 06B44702a from the New Energy and Industrial Technology Development Organization (NEDO) of Japan. 


\section{Notation}

$a_{\mathrm{m}}$ outer surface area of crystal, $\mathrm{m}^{2} \mathrm{~kg}^{-1}$

$C_{0}$ benzene concentration in cyclohexane solution at initial state, $\mathrm{mol} \mathrm{m}^{-3}$

$C_{\mathrm{t}}$ benzene concentration in cyclohexane solution at time $t, \mathrm{~mol} \mathrm{~m}^{-3}$

$C_{\mathrm{e}}$ benzene concentration in cyclohexane solution at equilibrium state, $\mathrm{mol} \mathrm{m}^{-3}$

$D_{\text {ad }}$ intracrystalline diffusivity in adsorption process, $\mathrm{m}^{2} \mathrm{~s}^{-1}$

$D_{\text {macro }}$ intracrystalline diffusivity within macro pore, $\mathrm{m}^{2} \mathrm{~s}^{-1}$

$H$ partition factor, dimensionless

$K$ slope of the Henry-type adsorption isotherm, $\mathrm{m}^{3} \mathrm{~kg}^{-1}$

$L$ half-thickness of the hexagonal slab shape material, $\mathrm{m}$

$M_{\mathrm{e}}$ amount adsorbed at adsorption equilibrium state, $\mathrm{mol} \mathrm{kg}^{-1}$

$M_{t}$ amount adsorbed at time $t$, mol $\mathrm{kg}^{-1}$

$q_{\mathrm{e}}$ amount adsorbed at equilibrium state, $\mathrm{mol} \mathrm{kg}^{-1}$

$r$ half size of secondary particle, $\mathrm{m}$

$R_{0}$ half size of the spherical shape material, $\mathrm{m}$

$t$ time, $\mathrm{s}$

$T$ temperature, $\mathrm{K}$

$W \quad$ adsorbent weight, $\mathrm{kg}$

Greek letter

$\rho$ density of porous material, $\mathrm{kg} \mathrm{m}^{-3}$ 


\section{References}

Crank, J., 1975. The Mathematics of Diffusion. Clarendon Press, Oxford.

Fujikata, Y., Masuda, T., Ikeda, H., Hashimoto, K., 1998. Measurement of the diffusivities within MFI- and Y-type zeolite catalysts in adsorption and desorption processes. Microporous and Mesoporous Materials, 21, 679-686

Hashimoto, K., 1979. Hanno-Kogaku. Baifu-kan.

Inagaki, S., Fukushima, Y., Kuroda, K., 1993. Synthesis of highly ordered mesoporous materials from a layered polysilicate. Journal of the Chemical Society-Chemical Communications, 8 , 680-682

Iwamoto, M., Tanaka, Y., Sawamura, N., Namba, S., 2003. Remarkable effect of pore size on the catalytic activity of mesoporous silica for the acetalization of cyclohexanone with methanol. Journal of the American Chemical Society, 125, $13032-13033$

Jama, M.A., Delmas, M.P.F., Ruthven, D.M., 1997. Diffusion of linear and branched $\mathrm{C}_{6}$ hydrocarbons in silicalite studied by the wall-coated capillary chromatographic method. Zeolites, 18, 200-204

Jobic, H., Bee, M., Caro, J., Bulow, M., Karger, J., 1989. Molecular self-diffusion of methane in zeolite ZSM-5 by quasielastic neutron-scattering and nuclear magneticresonance pulsed field gradient technique. Journal of the Chemical Society-Faraday Transactions I, 85, 4201-4209 
Kresge, C.T., Leonowicz, M.E., Roth, W.J., Vartuli, J.C., Beck, J.S., 1992. Ordered mesoporous molecular-sieves synthesized by a liquid-crystal template mechanism. Nature, 359, 710-712

Masuda, T., Fukada, K., Fujikata, Y., Ikeda, H., Hashimoto, K., 1996. Measurement and prediction of the diffusivity of Y-type zeolite. Chemical Engineering Science, 51, $1879-1888$

Masuda, T., Fujikata, Y., Nishida, T., Hashimoto, K., 1998. The influence of acid sites on intracrystalline diffusivties within MFI-type zeolites. Microporous and Mesoporous Materials, 23, 157-167

Masuda, T., Fujikata, Y., Ikeda, H., Hashimoto, K., 2000. Diffusivities in the binary components system within MFI-type zeolite crystals. Microporous and Mesoporous Materials, 38, 323-332

Masuda, T., Okubo, Y., Mukai, S.R., Kawase, M., Hashimoto, K., Shichi, A., Satsuma, A., Hattori, T., Kiyozumi, Y., 2001. Effective diffusivities of lighter hydrocarbons in $\mathrm{Cu}$ - and Co-MFI-type zeolite catalysts. Chemical Engineering Science, 56, 889-896

Masuda, T., 2003a. Diffusion mechanism of zeolite catalysis. Catalysis Survey from Asia, 7, $133-144$

Masuda, T., 2003b. Peculiar diffusion mechanisms within micropores of zeolite catalysts. Journal of the Japan Petroleum Institute, 46, 281-294

Masuda, T., Otani, S., Tsuji, T., Kitamura, M., Mukai, S.R., 2003c. Preparation of hydrophilic and acid-proof silicalite-1 zeolite membrane and its application to selective separation of water from water solution of concentrated acetic acid by pervaporation. Separation and Purification Technology, 32, 181-189 
Nakasaka, Y., Tago, T., Odate, K., Masuda, T., 2008. Measurement of intracrystalline diffusivity of benzene within MFI-type zeolite from bulk benzene/cyclohexane liquid phase. Microporous and Mesoporous Materials, 112, 162-169

Qizo, S.Z., Bhatia, S.K., 2005. Diffusion of $n$-decane in mesoporous MCM-41 silicas. Microporous and Mesoporous Materials, 86, 112-123

Shen, D.M., Rees, L.V.C., 1991. Diffusivities of benzene in HZSM-5, silicalite-1, and $\mathrm{NaX}$ determined by frequency-response techniques. Zeolites, 11, 666-671

Shah, D.B., Guo, D.J., Hayhurst, D.T., 1995. Intracrystalline diffusion of benzene in silicalite-effect of structural heterogeneity. Journal of the Chemical Society-Faraday Transactions, 91, 1143-1146

Song, L.J., Rees, L.V.C., 1996. Frequency response diffusion of propane in silicalite-1. Microporous Materials, 6, 363-374

Wilke, C.R., Chang, P., 1955. Correlation of diffusion coefficients in dilute solutions. AIChE Journal, 1, 264-270

Xiao, J.R., Wei, J., 1992, Diffusion mechanism of hydrocarbons in zeolites- 1. Theory. Chemical Engineering Science, 47, 1123-1141

Yanagisawa, T., Shimizu, T., Kuroda, K., Kato, C., 1990. Their conversion to microporous materials. Bulletin of the Chemical Society of Japan, 63, 988-992

Yano, K., Fukushima, Y., 2003. Particle size of mono-dispersed super-microporous silica spheres. Journal of Materials Chemistry, 13, 2577-2581

Yano, K., Fukushima, Y., 2004. Synthesis of mono-dispersed mesoporous silica spheres with highly ordered hexagonal regularity using conventional alkyltrimethylammonium halide as a surfactant. Journal of Materials Chemistry, 14, 1579-1584 
Yokoi, T., Yoshitake, H., Tatsumi, T., 2003. Synthesis of aminonic-surfactant-templated mesoporous silica using organoalkoxysilane-containig amino groups. Chemistry of Materials, 15, 4536-4538

Zikánová, A., Bülow, M., Schlodder, H., 1987. Intracrystalline diffusion of benzene in ZSM-5 and silicalite. Zeolites, 7, 115-118 


\section{Figures \& Table}

Table 1: Pore diameter of porous materials used in this work

Figure 1: SEM photographs of the silicalite-1, MMSS-10, MMSS-16, MMSS-18, and Silica Gel

Figure 2: Changes in the benzene concentrations with time

Figure 3: Effects of the pellet size of MMSS-16 on the benzene diffusivities

Figure 4: Adsorption isotherms of benzene onto silicalite-1, MMSS-10, MMSS-16, MMSS-18 and MMSS-22 at $323 \mathrm{~K}$

Figure 5: Effects of the amount of adsorbed benzene within MMSS materials on the intracrystalline diffusivity

Figure 6: Arrhenius plots of the intracrystalline diffusivity of benzene within micro, meso, and macro porous materials: the molecular diffusion coefficient was calculated by the Wilke-Chang equation (Wilke and Chang, 1955)

Figure 7: The relation between pore diameter, intracrystalline diffusivities, and activation energies

Figure 8: Liquid-phase diffusion models in (a) a configurational diffusion region, (b) a transition region, and (c) a molecular diffusion region 


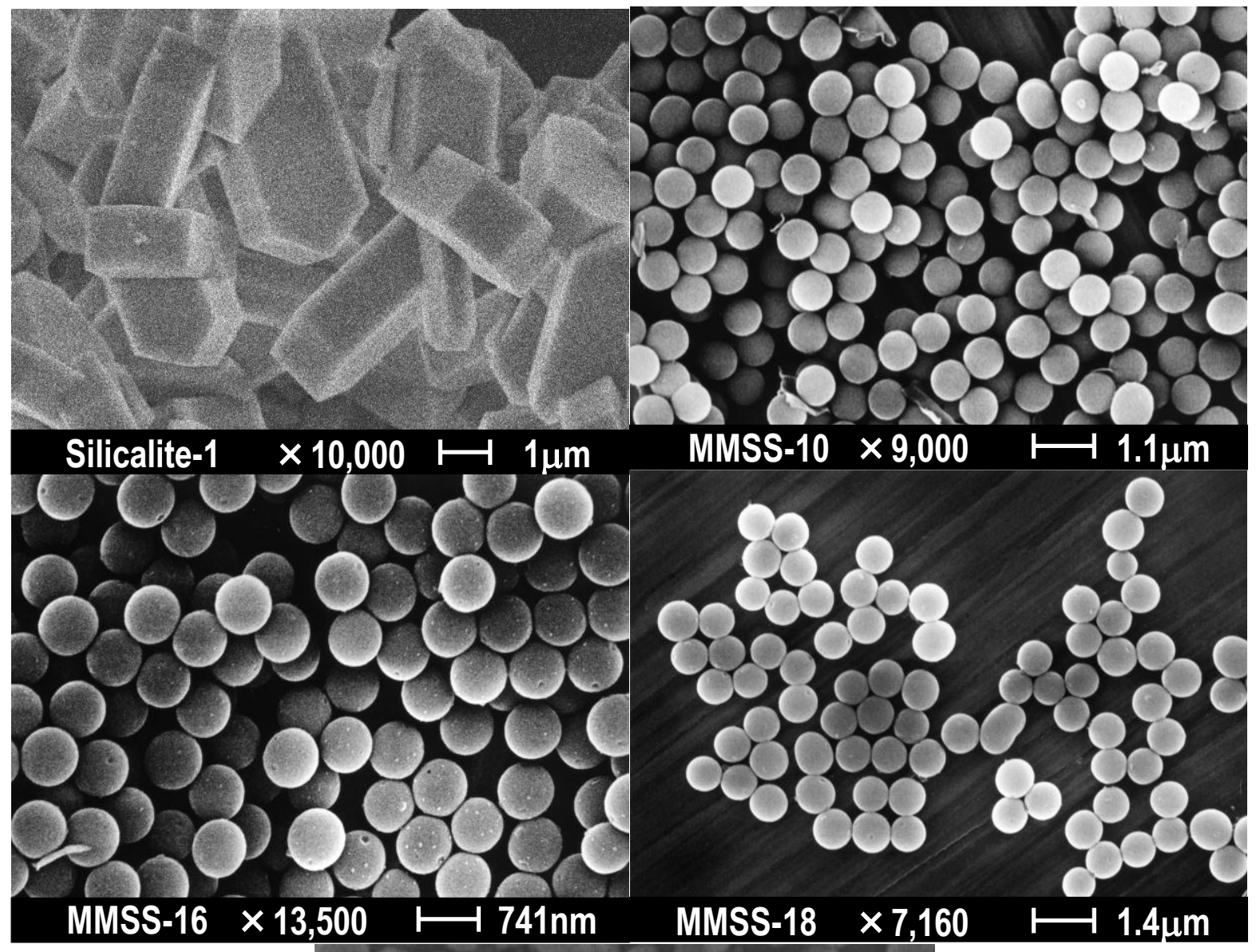

\section{Silica Gel $\times 1,500 \longmapsto 10 u m$}

Figure 1: SEM photographs of the silicallite-1, MMSS-10, MMSS-16, MMSS-18 and Silica Gel 


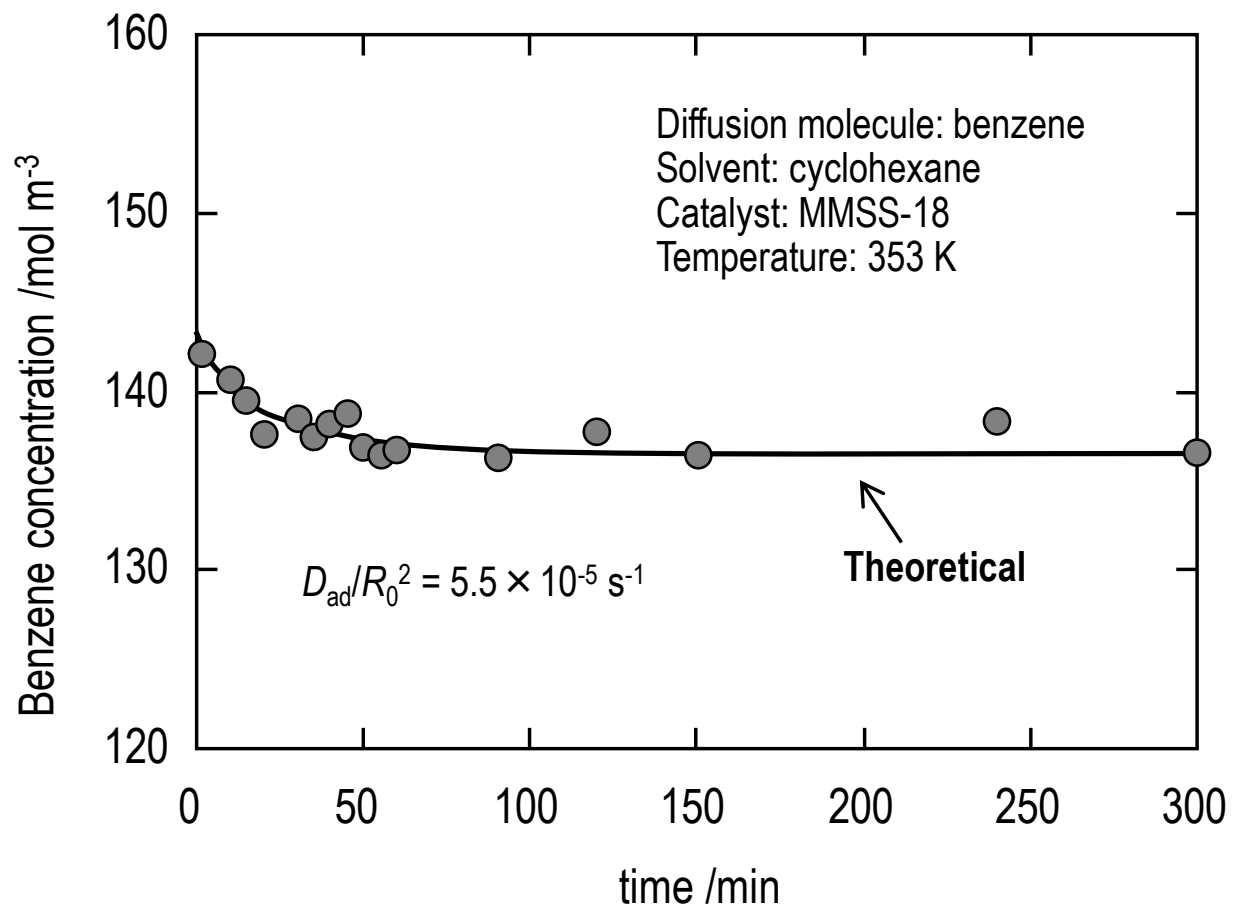

Figure 2: Changes in the benzene concentrations with time 


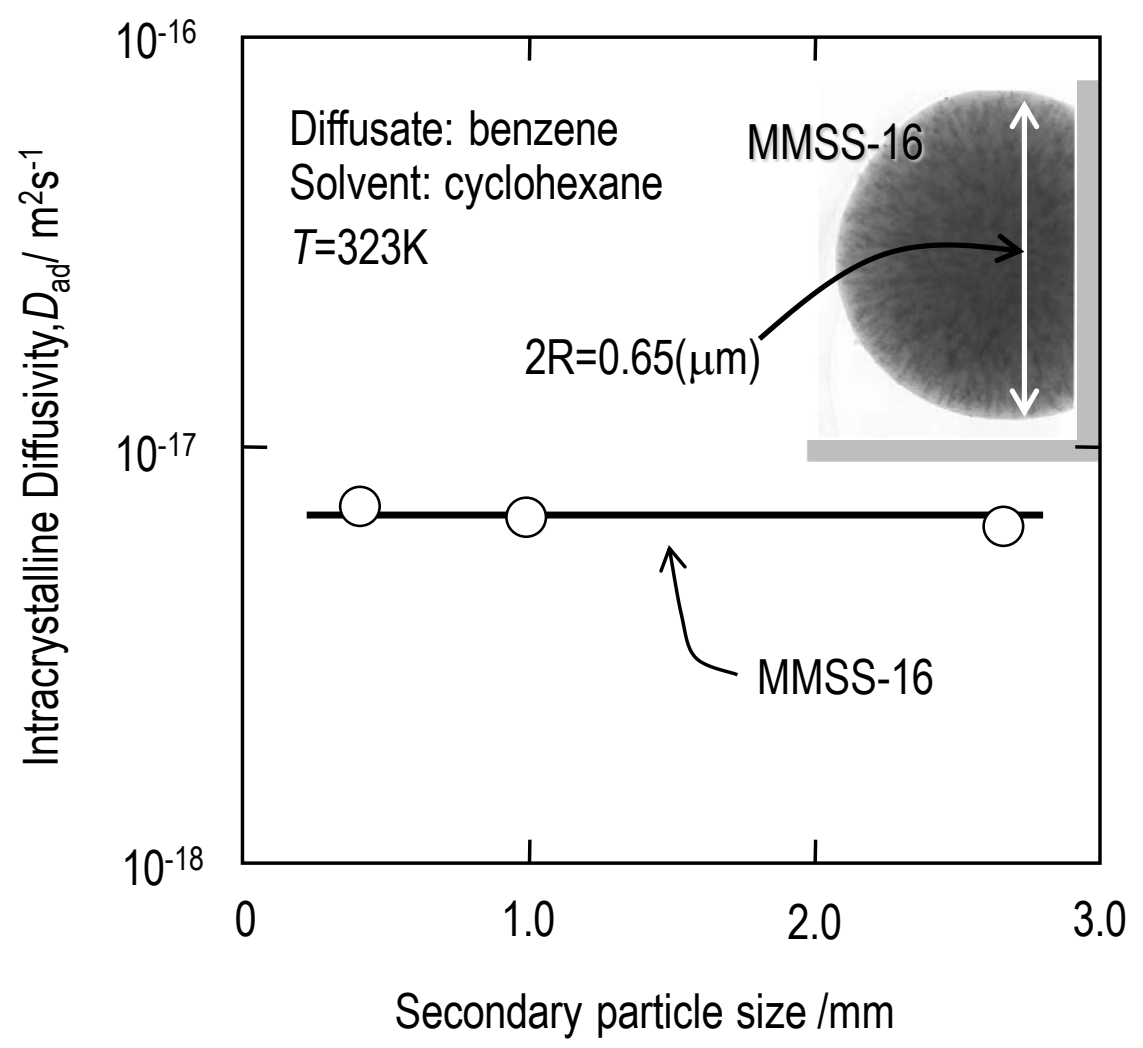

Figure 3: Effects of the pellet size of MMSS-16 on the benzene diffusivities 


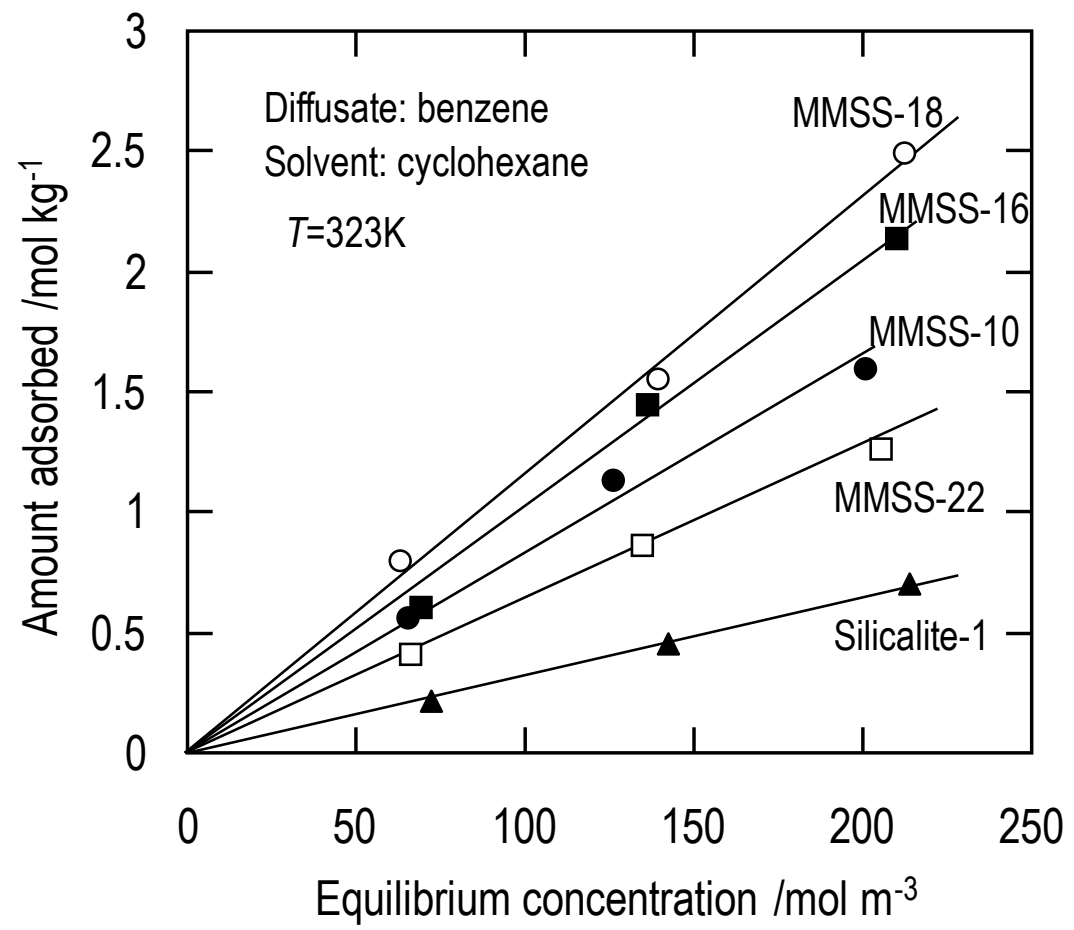

Figure 4: Adsorption isotherms of benzene onto silicalite-1, MMSS-10, MMSS-16, MMSS-18 and MMSS-22 at $323 \mathrm{~K}$ 


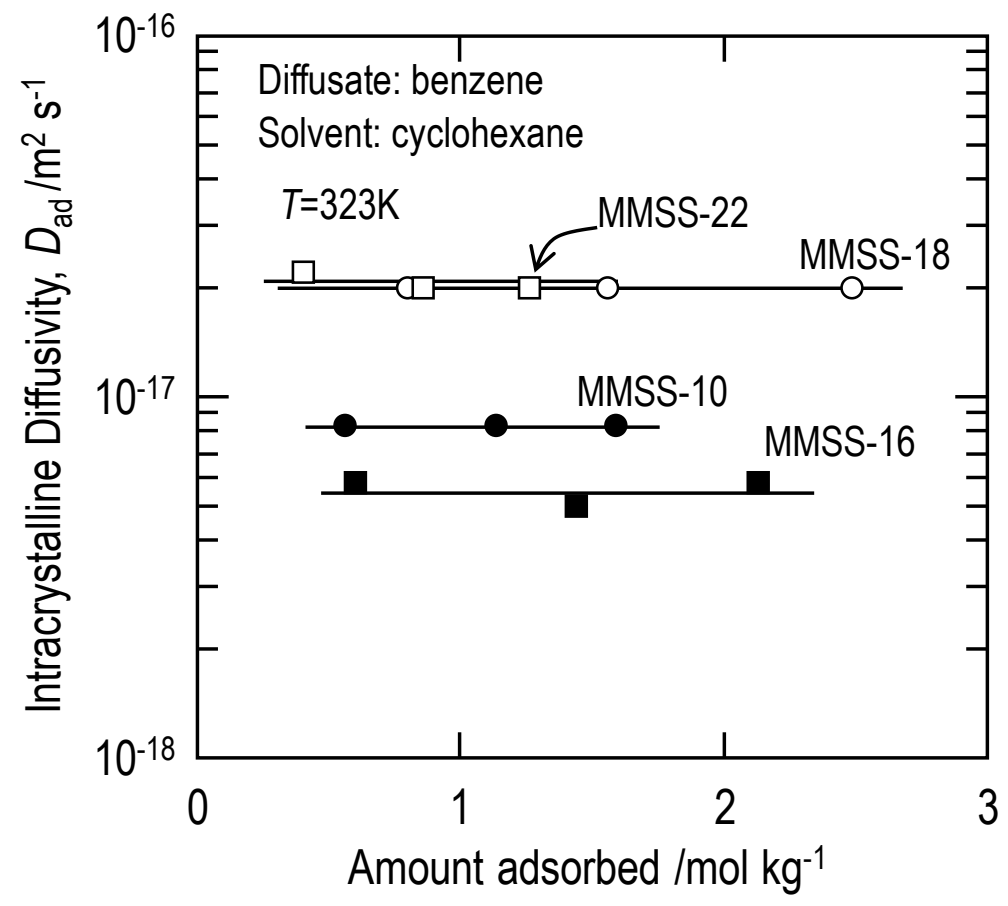

Figure 5: Effect of the amount adsorbed benzene within MMSS materials on the intracrystalline diffusivity 


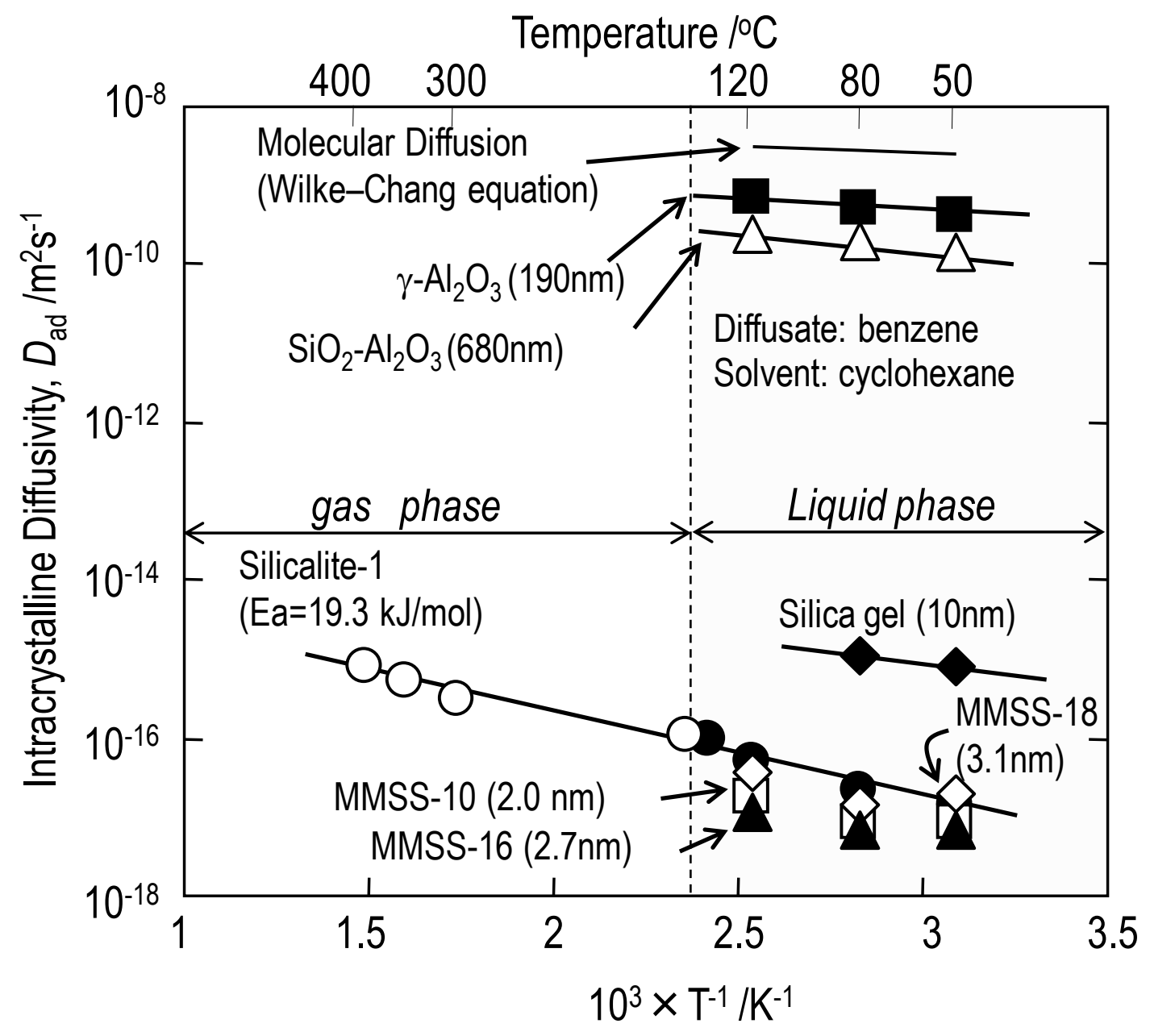

Figure 6: Arrhenius plots of the intracrystalline diffusivity of benzene within micro, meso and macro porous materials: the molecular diffusion coefficient was calculated by Wilke-Chang equation(Wilke and Chang, 1955) 


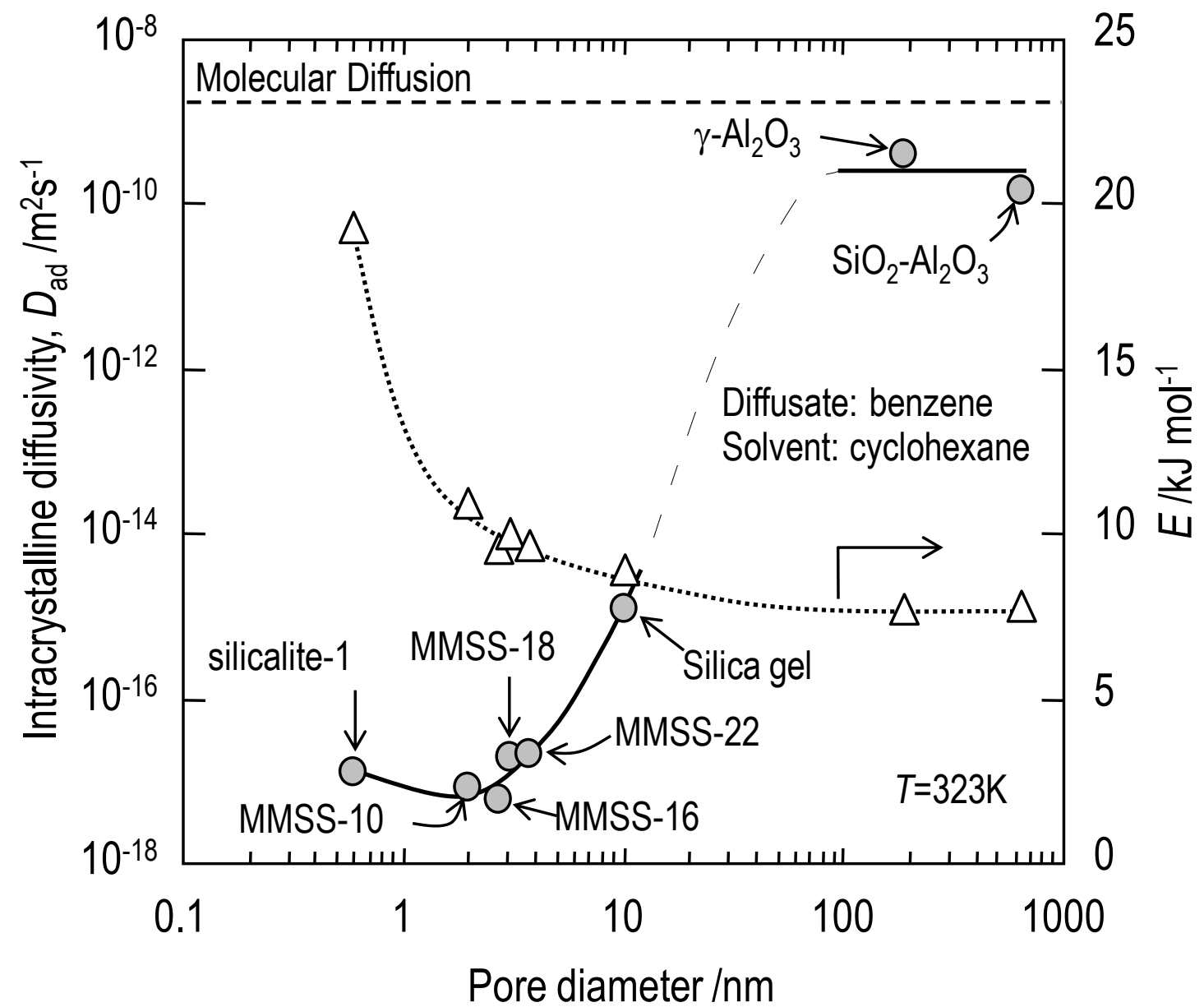

Figure 7: The relation between pore diameter and intracrystalline diffusivities, and activation energies 
(a) Configurational diffusion region bulk phase micro pore \{

\section{silicalite crystal}

discrete

(b) Transition region
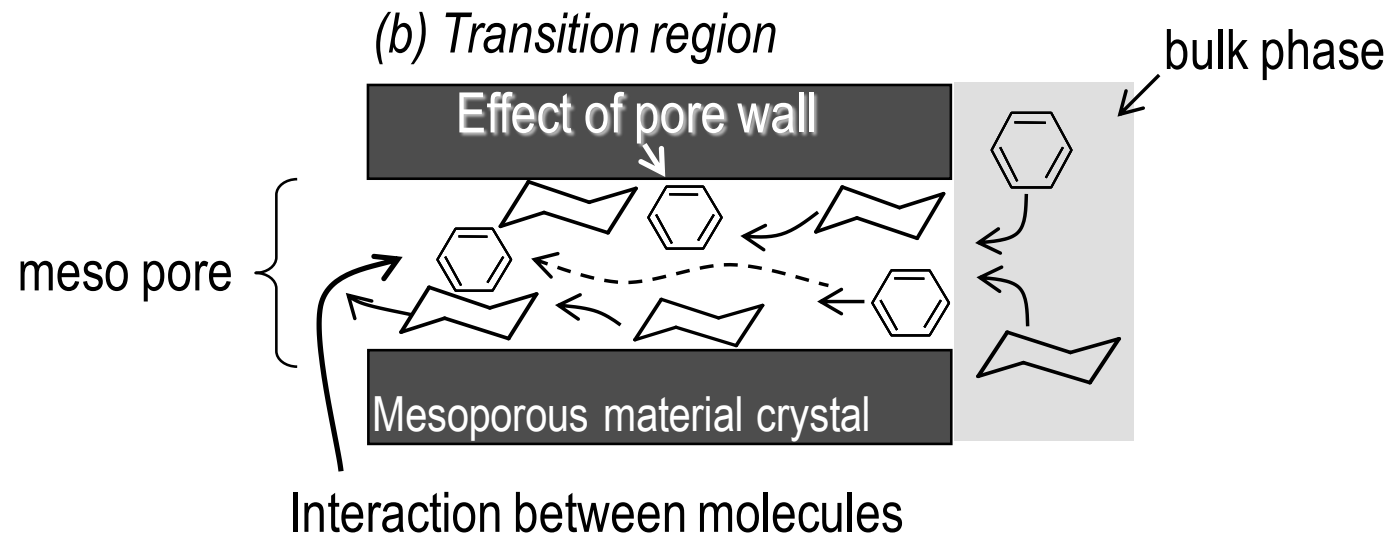

(c) Molecular diffusion region

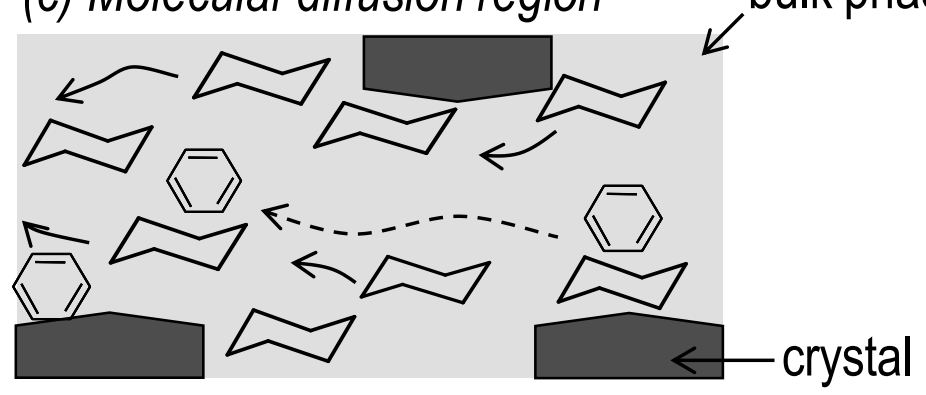

Figure 8: Liquid-phase diffusion models in (a) a configurational diffusion region, (b) a transition region, and (c) a molecular diffusion region 
Table 1 Pore diameter of porous materials used in this work

\begin{tabular}{cc}
\hline catalyst & pore diameter $(\mathrm{nm})$ \\
\hline silicalite-1 & 0.55 \\
MMSS-10 & 2 \\
MMSS-16 & 2.7 \\
MMSS-18 & 3.1 \\
MMSS-22 & 3.8 \\
Silica gel & 10 \\
$\gamma-\mathrm{Al}_{2} \mathrm{O}_{3}$ & 190 \\
$\mathrm{SiO}_{2}-\mathrm{Al}_{2} \mathrm{O}_{3}$ & 680 \\
\hline
\end{tabular}

